Internist 2020 $61: 416-423$

https://doi.org/10.1007/s00108-020-00773-5

Online publiziert: 16. März 2020

(c) Der/die Autor(en) 2020

\section{Redaktion}

H. Haller, Hannover (Schriftleitung)

B. Salzberger, Regensburg

C.C. Sieber, Nürnberg

\section{Anamnese}

Im April 2017 wurde ein 17-jähriger, männlicher Patient bei Verdacht auf eine rapid-progressive Glomerulonephritis (RPGN) in unsere nephrologische Abteilung übernommen. Anamnestisch fanden sich eine mehrtägige schmerzlose Makrohämaturie sowie im Vorfeld ein Infekt des oberen Respirationstrakts mit begleitenden Myalgien. Weiterhin klagte der Patient über intermittierende, stechende Schmerzen im Bereich der Brustwirbelsäule sowie über Dysurie. Hämoptysen, blutiger Schnupfen, Arthralgien oder Hautauffälligkeiten im Sinne eines Erythems bzw. Exanthems wurden verneint, ebenso chronische Vorerkrankungen, abgesehen von einem kindlichen Asthma bronchiale seit dem dritten Lebensjahr mit inhalativer Applikation von Salbutamol bei Bedarf. Seit etwa 3 Jahren bestand ein aktiver Nikotinkonsum (kumulativ etwa 1-2 Packungsjahre). Die Familienanamnese in Bezug auf Nierenerkrankungen war leer.

\section{Befund}

Bei Erstaufnahme in der zentralen Notaufnahme des peripheren Krankenhauses zeigte sich eine eingeschränkte Nierenfunktion (Kreatinin $1,36 \mathrm{mg} / \mathrm{dl}$, errechnete glomeruläre Filtrationsrate [eGFR] $74 \mathrm{ml} / \mathrm{min}$ ) ohne Sekundärkomplikationen, die sich im Verlauf weiter verschlechterte (eGFR $57 \mathrm{ml} / \mathrm{min}$ ). Im Spontanurin wurden eine (schmerzlose)

D. Chittka - L. Lennartz - B. Jung - B. Banas - T. Bergler

Abteilung Nephrologie, Universitätsklinikum Regensburg, Regensburg, Deutschland

\title{
Erfolgreiche Rituximabtherapie des Rezidivs einer Glomerulonephritis assoziiert mit Antikörpern gegen die glomeruläre Basalmembran
}

Makrohämaturie und große Albuminurie $(1,9 \mathrm{~g} / \mathrm{g}$ Kreatinin, Gesamtproteinurie 2,9g/g Kreatinin) nachgewiesen, sodass eine antiproteinurische Blockade des Renin-Angiotensin-AldosteronSystems (RAAS) mit Candesartan eingeleitet wurde. Ein Hinweis auf eine glomeruläre Hämaturie bestand in der auswärtigen Urinmikroskopie nicht. In der initialen auswärtigen Aufarbeitung mit Sonographie und kontrastmittelgestützter Computertomographie ergaben sich keine Erklärungen für die Makrohämaturie. In der rheumaserologischen Untersuchung mit Bestimmung der antinukleären Antikörper (ANA), zytoplasmatischen und perinukleären antineutrophilen zytoplasmatischen Antikörper (c-/p-ANCA), Antikörper gegen doppelsträngige DNA (Anti-dsDNA-AK), antimitochondrialen Antikörper (AMA), quantitativen Immunglobuline G, M und A (IgG, IgM, IgA), Komplementfaktoren C3c und C4 sowie Anti-Streptolysin-AK zeigte sich initial allein ein grenzwertig erhöhter ANA-Titer (1:160, gesprenkeltes Muster), die übrigen Parameter waren unauffällig. Schließlich ergab sich ein positiver Befund für die $\mathrm{AK}$ gegen die glomeruläre Basalmembran (Anti-GBM-AK; $78 \mathrm{U} / \mathrm{ml}$, Normbereich $<20 \mathrm{U} / \mathrm{ml}$ ), sodass der Patient in unsere universitäre nephrologische Abteilung verlegt wurde.

Laborchemisch zeigte sich eine progrediente Nierenfunktionseinschränkung (Kreatinin 1,6 mg/dl, eGFR nach der Chronic-Kidney-Disease-Epidemi-
ology-Collaboration-Formel $62 \mathrm{ml} / \mathrm{min}$ ) mit unauffälligen Ergebnissen für Elektrolyte und Harnstoff $(33 \mathrm{mg} / \mathrm{dl})$ sowie in der venösen Blutgasanalyse. Ferner waren das Blutbild und Differenzialblutbild unauffällig; sie lieferten keine Hinweise auf eine Hämolyse. Das Serumalbumin war mit 31,9g/l leicht erniedrigt, das C-reaktive Protein minimal erhöht. Bei anhaltend großer glomerulärer Proteinurie fielen in der mikroskopischen Untersuchung des Urinsediments als Änderung gegenüber dem externen Befund zahlreiche dysmorphe Erythrozyten (>50\%) und Akanthozyten (10-20\%) im Sinne einer glomerulären Hämaturie trotz fehlender Erythrozytenzylinder auf.

Bei hochgradigem Verdacht auf eine Anti-GBM-Glomerulonephritis wurde unmittelbar eine Plasmapheresebehandlung in Kombination mit intravenöser Steroidstoßtherapie (je $250 \mathrm{mg} \mathrm{Me}$ thylprednisolon i.v. über 3 Tage) eingeleitet.

\section{Nierenbiopsie}

In der am Folgetag durchgeführten Nierenbiopsie wiesen 3 der 17 getroffenen Glomeruli eine segmentale Basalmembranruptur mit fibrinoid-nekrotischem Material ohne Hinweis auf extrakapilläre Proliferation auf. Fokal stellte sich im Interstitium und peritubulär ein lympho-/monozytäres Infiltrat dar, einem geringgradigen akuten tubulointerstitiellen Schaden entsprechend. In der Immunfluoreszenzuntersuchung ergab sich der 
Hier steht eine Anzeige.

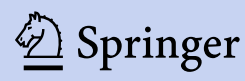




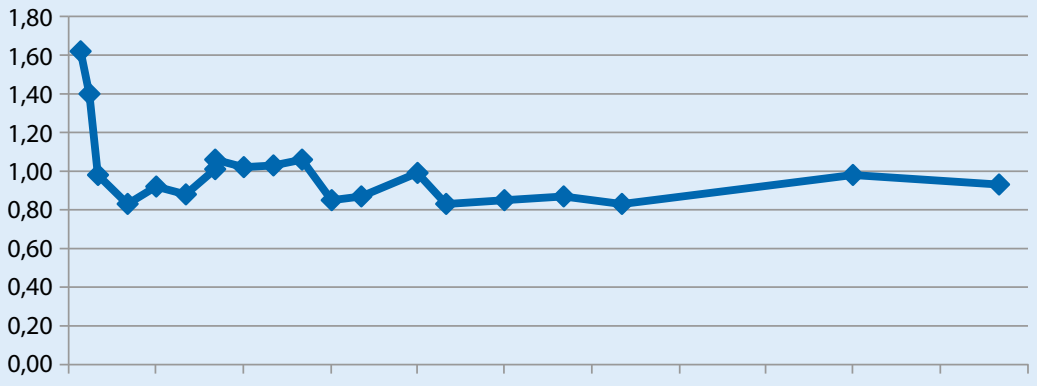

Apr 17 Jul 17 Okt 17 Jan 18 Apr 18 Jul 18 Okt 18 Jan 19 Apr 19 Jul 19 Okt 19

a

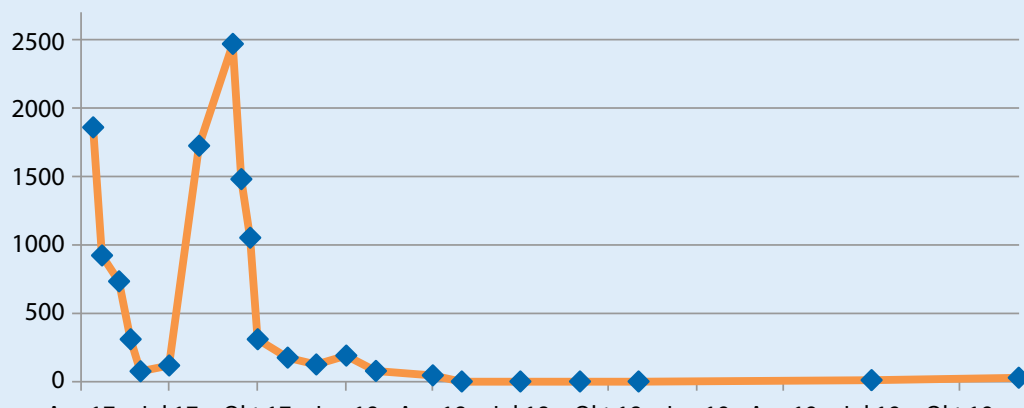

Apr 17 Jul 17 Okt 17 Jan 18 Apr 18 Jul 18 Okt 18 Jan 19 Apr 19 Jul 19 Okt 19

b
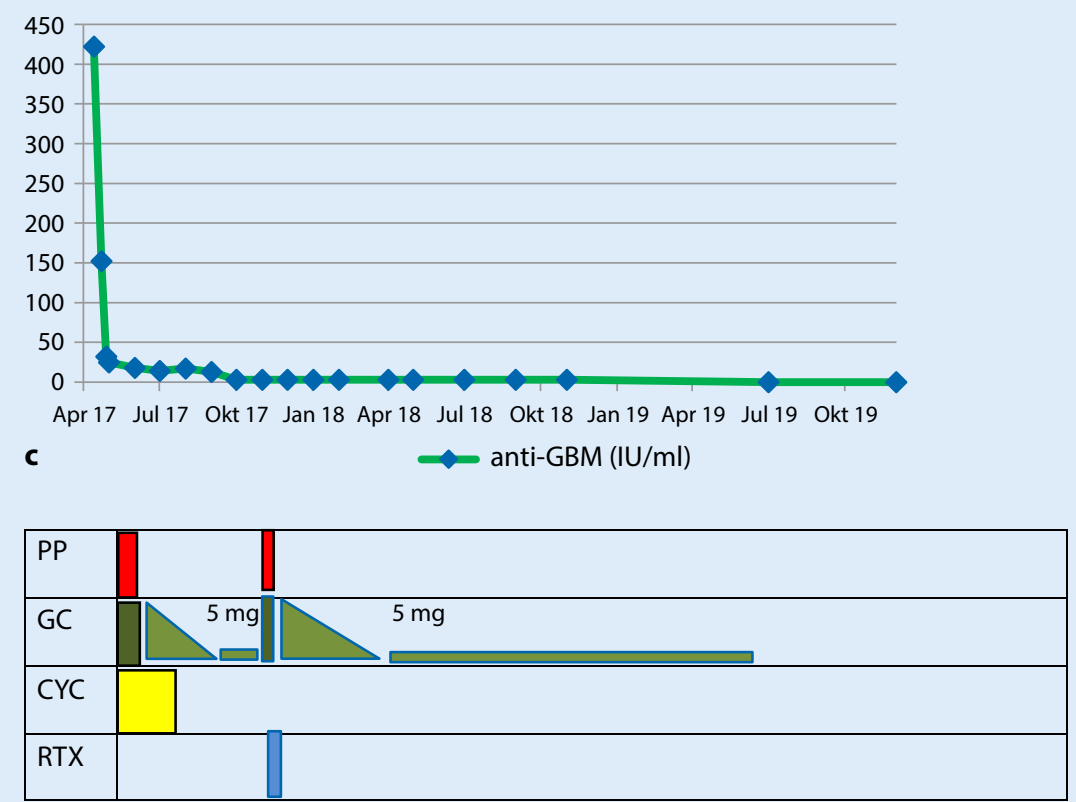

d
Abb. 1 - Verlauf von $\mathrm{SCr}(\mathrm{a})$, ACR im Spontanurin (b) und Anti-GBM-Antikörper-Titer (c) sowie Therapieverlauf (d). Nach Therapieeinleitung mit Plasmapherese, Steroiden sowie Cyclophosphamid waren Kreatinin, ACR und Anti-GB-

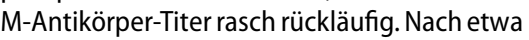
3 Monaten konnte die Therapie beendet werden. Etwa 2 Monate später zeigte sich erneut ein Anstieg der ACR, histologisch ergab sich der Nachweis eines Rezidivs der Anti-GBM-Glomerulonephritis. Die Anti-GBM-Antikörper sowie Kreatinin zeigten sich zu diesem Zeitpunkt unverändert. Nach erneuter Plasmapherese und 4-maliger RTX-Gabe war die ACR wieder regredient. Aktuell (32 Monate nach Erstdiagnose) liegen alle 3 Parameter im Normbereich (Kreatinin $0,93 \mathrm{mg} / \mathrm{dl}=$ errechnete glomeruläre Filtrationsrate $119 \mathrm{ml} / \mathrm{min}$, Anti-GBM $<2,9 \mathrm{IU} / \mathrm{ml}$, ACR $<50 \mathrm{mg} / \mathrm{g}$ Kreatinin). $A C R_{\text {" }}$ Albumin creatinine ratio" (Albumin-Kreatinin-Verhältnis); CYC Cyclophosphamid; GC Glukokortikoid; GBM glomeruläre Basalmembran; Krea Kreatinin;PPPlasmapherese, RTX Rituximab; SCr Serumkreatinin

Nachweis einer linearen Ablagerung von IgG entlang der GBM, sodass sich eindeutig die Diagnose einer Anti-GBMGlomerulonephritis mit fokal-segmentaler Nekrose ergab.

\section{Diagnose}

\section{- Anti-GBM-Glomerulonephritis mit fokal-segmentaler Nekrose}

\section{Therapie und Verlauf}

Parallel zur oben genannten Therapie wurde eine Therapie mit oralem Cyclophosphamid in einer Dosierung von $2 \mathrm{mg} / \mathrm{kgKG}$ pro Tag begonnen. Insgesamt wurden in einem Zeitraum von 14 Tagen 11 Plasmapheresesitzungen durchgeführt, mit Austausch gegen gefrorenes Frischplasma und einem durchschnittlich 1,5- bis 2-fach ausgetauschten Plasmavolumen pro Sitzung. Die Steroidtherapie wurde nach 3 Tagen auf eine orale Gabe umgestellt ( $1 \mathrm{mg} / \mathrm{kgKG}$ ) und im Verlauf bis zu einer Erhaltungstherapie von $5 \mathrm{mg} /$ Tag stufenweise reduziert.

Nach einer Therapiedauer von 2 Monaten waren Normwerte für Kreatinin und Anti-GBM-AK (18 IU/ml) sowie eine deutlich reduzierte Gesamtproteinurie bzw. Albuminurie zu verzeichnen (• Abb. 1a-c). Nach 3 Monaten (07/2017) wurde die Therapie mit oralem Cyclophosphamid (Kumulativdosis $6 \mathrm{~g}$ ) beendet und die Steroidtherapie mit einer Er- 
haltungsdosis von $5 \mathrm{mg}$ Prednisolon pro Tag fortgesetzt.

Nach klinisch sowie laborchemisch stabilem Verlauf im Rahmen der ambulanten Weiterbetreuung fiel 5 Monate nach Erstdiagnose (09/2017) eine deutliche Zunahme der Proteinurie und Albuminurie bei nur gering verschlechterter Nierenfunktion auf, zudem normwertige Anti-GBM-AK und ein fehlendes aktives Sediment. Der Patient war klinisch inapparent, allerdings mit fortgesetztem Nikotinkonsum.

Bei klinischem Verdacht auf ein Rezidiv der Grunderkrankung wurden erneut eine Plasmapheresetherapie und eine Erhöhung der Steroiddosis durchgeführt. Die wiederholte Nierenbiopsie zeigte erneut eine lineare IgG-Ablagerung - ohne akute Nekrose, jedoch mit einer ausgeprägten fibrozellulären, extrakapillären Reaktion, was eine kürzlich abgelaufene Nekrose vermuten ließ. Nachdem es unter Plasmapherese bereits zu einem Rückgang der Albuminurie gekommen war, entschied man sich nach deren Ende zu einer immunsuppressiven Therapie mit Rituximab (RTX) i.v. ( $375 \mathrm{mg} / \mathrm{m}^{2}$ Körperoberfläche; 4 Einzeldosen à $900 \mathrm{mg}$ im Abstand von einer Woche). Zusätzlich wurde wegen der diskrepanten Befunde (negative AK-Serologie, aber positiver histologischer Befund) eine indirekte Immunfluoreszenzuntersuchung an Affennieren als sensitivere Bestimmungsmethode durchgeführt. Hier ergab sich im Gegensatz zum negativen „enzymelinked immunosorbent assay“" (ELISA) ein positiver Befund für Anti-GBM-AK.

Unter oben genanntem Therapieschema gingen die Gesamtproteinurie und Albuminurie weiter zurück, sodass zuletzt (12/2019, 32 Monate nach Erstdiagnose) Normwerte dokumentiert werden konnten (Proteinurie $<50 \mathrm{mg} / \mathrm{g}$ Kreatinin, Albuminurie $<5 \mathrm{mg} / \mathrm{g}$ Kreatinin, Kreatinin 0,93 mg/dl, eGFR $119 \mathrm{ml} / \mathrm{min}$; - Tab. 1). Die Therapie mit Prednisolon wurde im Februar 2019 beendet.

\section{Diskussion}

Die Anti-GBM-Erkrankung (vormals Goodpasture-Syndrom) ist ein sehr seltenes Krankheitsbild aus dem Formenkreis der Kleingefäßvaskulitiden

Internist 2020 -61:416-423 https://doi.org/10.1007/s00108-020-00773-5

(c) Der/die Autor(en) 2020

D. Chittka · L. Lennartz · B. Jung · B. Banas · T. Bergler

\section{Erfolgreiche Rituximabtherapie des Rezidivs einer Glomerulonephritis assoziiert mit Antikörpern gegen die glomeruläre Basalmembran}

\section{Zusammenfassung}

Es wird über die erfolgreiche Behandlung des Rezidivs einer Anti-GBM-Erkrankung (assoziiert mit Antikörpern gegen die glomeruläre Basalmembran [GBM]) mittels Rituximab bei einem 17-jährigen Patienten berichtet. Die Nierenbiopsie mit Nachweis einer linearen Immunglobulin-G-Ablagerung entlang der Basalmembran stellt den Goldstandard dar, der von serologischen Analysen begleitet wird. Standardassays zur Anti-GBM-Bestimmung weisen hohe
Raten an falsch-negativen Befunden auf. Eine Zunahme der Proteinurie trotz Standardtherapie mit Plasmapherese, Steroid und Cyclophosphamid war das klinische Korrelat des Erkrankungsrezidivs. Rituximab führte zu einer vollständigen Ausheilung.

\section{Schlüsselwörter}

Rezidiv der Anti-GBM-Erkrankung · Akutes Nierenversagen · Nierenbiopsie $\cdot$ Monoklonale Antikörper · Plasmaaustausch

\section{Successful rituximab treatment of recurrent glomerulonephritis associated with antibodies against the glomerular basement membrane}

\begin{abstract}
This article presents a case of recurrent antiGBM disease (with antibodies against the glomerular basement membrane [GBM]) in a 17-year-old patient successfully treated with rituximab. Kidney biopsy with detection of linear deposition of immunoglobulin $G$ ( $\operatorname{lgG}$ ) along the basement membrane is the diagnostic gold standard, which should be accompanied by serological testing. However, standard assays for the detection of anti-GBM antibodies have a high rate of false-negative
\end{abstract}

results. In this particular case, an increase in proteinuria despite standard therapy (plasmapheresis, steroids, cyclophosphamide) was the clinical correlate of relapsing disease. The use of rituximab completely resolved the recurrent anti-GBM disease.

Keywords Anti-GBM disease, relapse - Acute kidney injury $\cdot$ Kidney biopsy . Antibodies, monoclonal . Plasma exchange mit einer Inzidenz von 1 bis 2 Fällen pro 1 Mio. Einwohner in europäischen Kohorten [1]. Die pathogenetischen Autoantikörper binden typischerweise an die NC1-Domäne der a3-Kette von Kollagen Typ IV, das in der Basalmembran glomerulärer und pulmonaler Kapillaren exprimiert ist. Daher sind RPGN und alveoläre Hämorrhagie die typischen klinischen Manifestationen dieser Autoimmunerkrankung [2]. Zum Zeitpunkt der Diagnose zeigt der überwiegende Teil der Patienten Symptome einer RPGN, aber nur etwa 50\% weisen Zeichen einer alveolären Schädigung auf $[2,3]$. Nur ein sehr geringer Prozentsatz zeigt bei Erstmanifestation eine isolierte alveoläre Hämorrhagie [3]. Als potenzielle Trigger für die Erstmanifestation der
Vaskulitis werden respiratorische Infekte diskutiert.

\section{Diagnostik}

Die Diagnosesicherung beinhaltet neben Urinanalysen die serologische Testung auf Anti-GBM-IgG-AK sowie die zeitnahe Nierenpunktion. Bezüglich der serologischen Testverfahren ist $\mathrm{zu}$ beachten, dass die konventionell verfügbaren Nachweismethoden, beispielsweise ELISA, in bis zu $36 \%$ der Fälle falschnegative Ergebnisse erbringen [4]. Sensitivere Methoden, wie ein Biosensorassay oder eine indirekte Immunfluoreszenzuntersuchung an Affennieren, stehen in Speziallaboren zur Verfügung und sollten bei Patienten mit anhaltendem 


\section{Kasuistiken}

Tab. 1 Zeitlicher Verlauf der laborchemischen und klinischen Parameter

\begin{tabular}{|c|c|c|c|c|c|c|}
\hline & $\begin{array}{l}\text { Serum- } \\
\text { kreatinin } \\
\text { (mg/dl) }\end{array}$ & $\begin{array}{l}\text { Errechnete glome- } \\
\text { ruläre Filtrationsra- } \\
\text { te ( } \mathrm{ml} / \mathrm{min})\end{array}$ & $\begin{array}{l}\text { Anti-GBM- } \\
\text { Antikörper } \\
(\mathrm{IU} / \mathrm{ml})\end{array}$ & $\begin{array}{l}\text { Albumin-Kreatinin- } \\
\text { Verhältnis (mg/g } \\
\text { Kreatinin) }\end{array}$ & $\begin{array}{l}\text { Glomeru- } \\
\text { läre Hä- } \\
\text { maturie }\end{array}$ & Histologischer Befund \\
\hline $\begin{array}{l}\text { Erstaufnahme } \\
\text { (04/2017) }\end{array}$ & 1,6 & 62 & 422 & 1859 & Ja & $\begin{array}{l}\text { Anti-GBM-GN mit fokal-segmentaler } \\
\text { Nekrose ( } 3 / 17 \text { Glomeruli) }\end{array}$ \\
\hline $\begin{array}{l}2 \text { Monate nach } \\
\text { Erstdiagnose } \\
(06 / 2017)\end{array}$ & 0,83 & 129 & 18 & 310 & Nein & - \\
\hline $\begin{array}{l}\text { Wiederaufnahme } \\
\text { (09/2017) = Rezi- } \\
\text { div }\end{array}$ & 1,1 & 98 & 16,3 & 2467 & Nein & $\begin{array}{l}\text { Anti-GBM-GN mit ausgeprägter } \\
\text { fibrozellulärer, extrakapillärer Reakti- } \\
\text { on ( } 2 / 16 \text { Glomeruli) }\end{array}$ \\
\hline $\begin{array}{l}\text { Letzte Verlaufs- } \\
\text { untersuchung } \\
\text { (12/2019) }\end{array}$ & 0,93 & 119 & $<2,9$ & $<50$ & Nein & - \\
\hline
\end{tabular}

klinischem Verdacht und negativem Befund im Screeningassay in Erwägung gezogen werden [5]. Andere Ursachen einer RPGN sollten im Rahmen eines rheumatologischen Screenings (ANA, c/p-ANCA, C3, C4, dsDNA-AK) stets parallel mit untersucht werden. Oftmals besteht eine Überschneidung der AntiGBM-Erkrankung mit den ANCA-assoziierten Vaskulitiden; in bis zu 10-50\% der Fälle weisen Patienten mit AntiGBM-Erkrankung auch ANCA auf, wobei es sich zu fast $100 \%$ um p-ANCA gegen die Myeloperoxidase handelt [6].

\section{》) Eine lineare IgG-Ablagerung entlang der glomerulären Basal- membran ist pathognomonisch}

Eine Nierenbiopsie sollte bei klinischem Verdacht und laborchemischen Zeichen einer rasch verlaufenden Nierenschädigung so früh wie möglich durchgeführt und keinesfalls durch ausstehende oder negative serologische Befunde verzögert werden [2]. In der histologischen Aufarbeitung mit Immunfluoreszenzuntersuchung findet sich pathognomonisch eine lineare Ablagerung von IgG entlang der GBM. Zudem lassen sich bei $>95 \%$ der Patienten Nekrosen mit Halbmondbildung nachweisen. Der Anteil nekrotisch veränderter Glomeruli korreliert eng mit dem Ausmaß der Nierenschädigung zum Diagnosezeitpunkt und ist ein wichtiger prognostischer Faktor für das spätere renale Outcome [7].
Bei klinischem Verdacht auf eine pulmonale Beteiligung dienen die hochauflösende Computertomographie (HRCT) und die bronchoalveoläre Lavage als diagnostische Methoden. Vor allem bei Patienten mit begleitender ANCA-Positivität sollte eine HRCT der Lunge und gegebenenfalls der Nasennebenhöhlen zum Nachweis der ANCA-Vaskulitistypischen Veränderungen erfolgen.

\section{Therapie}

Therapieprotokolle sehen die Plasmaseparation mit anschließender immunsuppressiver Therapie vor, typischerweise mit einer Kombination aus oralem Cyclophosphamid (2 mg/kgKG) und Kortikosteroiden. Der Stellenwert einer initialen Steroidstoßtherapie ist nicht durch die Studienlage zu belegen [7].

Laut aktuellen Empfehlungen erfolgt eine initiale Plasmaseparationstherapie über 2-3 Wochen, wenn möglich initial täglich. Danach sollte anhand der AK-Titer und des Verlaufs der Nierenfunktion sowie gegebenenfalls der Lungenbeteiligung die Indikation für eine Fortführung des Plasmaaustauschs geprüft werden. Die optimale Dauer der immunsuppressiven Therapie ist nicht bekannt. Basierend auf Studienergebnissen wird meist eine Gesamttherapiedauer von 2 bis 3 Monaten empfohlen, falls die AntiGBM-AK, die regelmäßig kontrolliert werden müssen, negativ bleiben [7]. Eine spezifische Therapie zur Remissionserhaltung wird nicht empfohlen. Bei fehlendem Ansprechen wird der Einsatz von Azathioprin (1-2 mg/kg pro Tag) oder Mycophenolatmofetil (1000 mg 2-mal täglich) für 6-9 Monate diskutiert [8].

In einer Kohorte von 72 Patienten mit Anti-GBM-Glomerulonephritis, die alle wie oben angegeben behandelt wurden, konnte eine dialysefreie Erhaltung der Nierenfunktion in $>90 \%$ der Fälle erreicht werden [7]. Dabei zeigt sich, dass die Dialysenotwendigkeit bei Erstdiagnose, der Anteil an gesunden Glomeruli in der Biopsie und das Ausmaß einer tubulointerstitiellen Schädigung als Prädiktoren für die Entwicklung einer terminalen Niereninsuffizienz im Verlauf dienen können [9]. Ein Krankheitsrezidiv bei isoliert anti-GBM-positiven Patienten wird als sehr seltenes Ereignis beschrieben $(<3 \%$ in den größten Studienpopulationen) und scheint mit fortgesetztem Nikotinkonsum assoziiert zu sein [10].

In unserem Fall konnte unter oben genannter Standardtherapie ein gutes Sofortansprechen erzielt werden, jedoch zeigte sich bereits 2 Monate nach Ende der Cyclophosphamidtherapie ein Rezidiv der Anti-GBM-Erkrankung. Es fiel die Entscheidung zur erneuten Plasmapheresebehandlung sowie zur Einleitung einer immunsuppressiven Therapie basierend auf RTX.

RTX ist ein Anti-CD20-AK, der zur Depletion aller CD20-positiven B-Zellen führt. In den letzten Jahren konnte sich RTX unter anderem in der Therapie rheumatologischer Erkrankungen, wie der rheumatoiden Arthritis, aber 
auch bei den c-/p-ANCA-assoziierten Vaskulitiden etablieren. Bei der AntiGBM-Glomerulonephritis ist eine Therapie mit RTX bisher nur in Einzelfällen beschrieben. In der größten retrospektiven Untersuchung von 8 Patienten mit therapierefraktärem Rezidiv einer Anti-GBM-Erkrankung (6 ohne und 2 mit pulmonaler Beteiligung) konnten unter Einsatz von RTX bei 7 von $8 \mathrm{~Pa}$ tienten eine klinische Remission und negative Anti-GBM-AK-Spiegel, jedoch keine Verbesserung der Nierenfunktion erreicht werden [11]. In einem anderen Fall konnte bei einem Rezidiv des Goodpasture-Syndroms und fehlendem Ansprechen auf eine erneute Cyclophosphamidtherapie unter RTX schließlich eine Remission erreicht werden [12].

\section{》) Bei der Anti-GBM- Glomerulonephritis ist eine Rituximabtherapie nur in Einzelfällen beschrieben}

Auch bei unserem Patienten zeigte sich nach erneuter Plasmapheresebehandlung sowie RTX-Applikation in oben genannter Dosierung die Proteinurie wieder rasch regredient. In der Nachbeobachtung waren die Nierenfunktion sowie die Proteinurie stabil und der AntiGBM-Titer anhaltend negativ. Alternativ zu dem hier vorgestellten Therapieschema gibt es auch einzelne Fallserien in der Literatur, in denen RTX bereits als Induktionstherapie allein oder in Kombination mit Cyclophosphamid eingesetzt wurde und gute Ergebnisse erzielt werden konnten [13, 14]. Aufgrund der niedrigen Fallzahl hat dies jedoch noch keinen Einzug in die offiziellen Therapieempfehlungen gefunden, sollte jedoch im Einzelfall diskutiert werden.

\section{Resümee}

Zusammenfassend veranschaulicht dieser Fall, dass die Anti-GBM-Glomerulonephritis bereits in jungen Jahren auftreten kann, die Nierenbiopsie ganz klar der diagnostische Goldstandard ist, die glomeruläre Proteinurie einen wertvollen klinischen Verlaufsmarker darstellt und Rezidive trotz leitliniengerechter Immunsuppression auch im kurzfristigen Verlauf möglich sind. RTX könnte für Rezidive unter bzw. nach Cyclophosphamidtherapie an Bedeutung gewinnen.

\section{Fazit für die Praxis}

- Bei gleichzeitigem Auftreten von akutem Nierenversagen mit Hinweisen auf eine rapid-progressive Glomerulonephritis und alveolären Hämoptysen muss an eine Glomerulonephritis mit Antikörpern gegen die glomeruläre Basalmembran (GBM) gedacht werden.

- Die Nierenbiopsie stellt den diagnostischen Goldstandard dar und sollte nicht durch andere diagnostische Verfahren verzögert werden.

- Die serologischen Tests auf Anti-GBMAntikörper sind anfällig für falschnegative Ergebnisse und sollten bei begründetem klinischem Verdacht durch sensitivere Tests erweitert werden.

- Rezidive der Erkrankung sind trotz adäquater Therapie möglich.

- Die Therapie mit Rituximab kann ein Ansatz bei konservativ nicht beherrschbaren Verläufen sein.

\begin{tabular}{l} 
Korrespondenzadresse \\
$\begin{array}{l}\text { Abteilung Nephrologie, } \\
\text { Universitätsklinikum } \\
\text { Regensburg } \\
\text { Franz-Josef-Strauß-Allee 11, } \\
\text { 93053 Regensburg, } \\
\text { Deutschland } \\
\text { dominik.chittka@ } \\
\text { klinik.uni-regensburg.de }\end{array}$ \\
\hline
\end{tabular}

Funding. Open Access funding provided by Projekt DEAL.

\section{Einhaltung ethischer Richtlinien}

Interessenkonflikt. D. Chittka, L. Lennartz, B. Jung, B. Banas und T. Bergler geben an, dass kein Interessenkonflikt besteht.

Für diesen Beitrag wurden von den Autoren keine Studien an Menschen oder Tieren durchgeführt. Für die aufgeführten Studien gelten die jeweils dort angegebenen ethischen Richtlinien. Für Bildmaterial oder anderweitige Angaben innerhalb des Manuskripts, über die Patienten zu identifizieren sind, liegt von innen und/oder ihren gesetzlichen Vertretern eine schriftliche Einwilligung vor.

Open Access Dieser Artikel wird unter der Creative Commons Namensnennung 4.0 International Lizenz veröffentlicht, welche die Nutzung, Vervielfältigung, Bearbeitung, Verbreitung und Wiedergabe in jeglichem Medium und Format erlaubt, sofern Sie den/die ursprünglichen Autor(en) und die Quelle ordnungsgemäß nennen, einen Link zur Creative Commons Lizenz beifügen und angeben, ob Änderungen vorgenommen wurden.

Die in diesem Artikel enthaltenen Bilder und sonstiges Drittmaterial unterliegen ebenfalls der genannten Creative Commons Lizenz, sofern sich aus der Abbildungslegende nichts anderes ergibt. Sofern das betreffende Material nicht unter der genannten Creative Commons Lizenz steht und die betreffende Handlung nicht nach gesetzlichen Vorschriften erlaubt ist, ist für die oben aufgeführten Weiterverwendungen des Materials die Einwilligung des jeweiligen Rechteinhabers einzuholen.

Weitere Details zur Lizenz entnehmen Sie bitte der Lizenzinformation auf http://creativecommons.org/ licenses/by/4.0/deed.de.

\section{Literatur}

1. Canney M, O'Hara PV, McEvoy CM, Medani S, Connaughton DM, Abdalla AA, Doyle R, Stack AG, O'Seaghdha CM, Clarkson MR, Griffin MD, Holian J, Dorman AM, Niland A, Keogan M, Wallace EM, Conlon NP, Walsh C, Kelly A, Little MA (2016) Spatial and temporal clustering of anti-glomerular basement membrane disease. Clin J Am Soc Nephrol 11:1392

2. McAdoo SP, Pusey CD (2017) Anti-glomerular basement membrane disease. Clin J Am Soc Nephrol 12:1162

3. Lazor R, Bigay-Gamé L, Cottin V, Cadranel J, Decaux O, Fellrath J-M, Cordier J-F (2007) Alveolarhemorrhage in anti-basementmembrane antibody disease: a series of 28 cases. Medicine 86:181

4. Sinico RA, Radice A, Corace C, Sabadini E, Bollini B (2006) Anti-glomerular basement membrane antibodies in the diagnosis of Goodpasture syndrome: a comparison of different assays. Nephrol Dial Transplant 21:397

5. Sadeghi-Alavijeh O, Henderson S, Bass P, Cook T, DeGroot K, Salama AD (2018) Crescentic glomerulonephritis with anti-GBM antibody but no glomerular deposition. BMC Nephrol 19:228

6. McAdooSP, Tanna A, HruškováZ, Holm L, WeinerM, Arulkumaran N, Kang A, Satrapová V, Levy J, Ohlsson S, Tesar V, Segelmark M, Pusey CD (2017) Patients double-seropositive for ANCA and anti-GBM antibodies have varied renal survival, frequency of relapse, and outcomes compared to single-seropositive patients. Kidney Int 92:693

7. Levy JB, Turner AN, Rees AJ, Pusey CD (2001) Long-term outcome of anti-glomerular basement membrane antibody disease treated with plasma exchange and immunosuppression. Ann Intern Med 134:1033

8. UpToDate. http://www.uptodate.com

9. van Daalen EE, Jennette JC, McAdoo SP, Pusey CD, Alba MA, Poulton CJ, Wolterbeek R, Nguyen TQ, Goldschmeding R, Alchi B, Griffiths M, de 
Zoysa JR, Vincent B, Bruijn JA, Bajema IM (2018) Predicting outcome in patients with anti-GBM glomerulonephritis. Clin JAm Soc Nephrol 13:63

10. Gu B, Magil AB, Barbour SJ (2016) Frequently relapsing anti-glomerular basement membrane antibody disease with changing clinical phenotype and antibody characteristics over time. Clin Kidney J 9:661

11. Touzot $M$, Poisson J, Faguer $S$, Ribes $D$, Cohen $P$, Geffray L, Anquel N, François H, Karras A, Cacoub P, Durrbach A, Saadoun D (2015) Rituximab in antiGBM disease: A retrospective study of 8 patients. JAutoimmun 60:74

12. Arzoo K, Sadeghi S, Liebman HA (2002) Treatment of refractory antibody mediated autoimmune disorders with an anti-CD20 monoclonal antibody (rituximab). Ann Rheum Dis 61:922

13. Syeda UA, Singer NG, Magrey M (2013) Antiglomerularbasementmembraneantibodydisease treated with rituximab: a case-based review. Semin Arthritis Rheum 42(6):567-572

14. Heitz M, Carron PL, Clavarino G, Jouve T, Pinel N, Guebre-Egziabher F, Rostaing L (2018) Use of rituximab as an induction therapy in antiglomerular basement-membrane disease. BMC Nephrol 19(1):241

\section{SARS-CoV-2: Was Rheuma-Patienten wissen sollten}

Relevante Beeinträchtigung der Immunfunktion?

Patienten mit rheumatischen Erkrankungen und Patienten unter immunsuppressiver Therapie könnten ebenfalls ein besonderes Risiko für einen schweren Verlauf einer SARS-CoV-2-Infektion haben, wie die Deutsche Gesellschaft für Rheumatologie e.V. (DGRh) mitteilt. Ob dies tatsächlich der Fall ist, lässt sich nicht sicher sagen, da es bisher keine Daten dazu gibt. Menschen mit Rheuma sollten sich deshalb sehr konsequent vor einer Infektion schützen, indem sie die empfohlenen Maßnahmen des RKI sorgfältig beachten.

Derzeit gibt es keine belastbaren Zahlen da$\mathrm{zu}$, ob - und wenn wie - sich eine entzündlich-rheumatische Vorerkrankung auf den Verlauf einer SARS-CoV-2-Infektion auswirkt. „Wir wissen aber, dass Patienten mit aktivem Rheuma generell infektanfälliger sind", gibt Prof. Dr. med. Hendrik Schulze-Koops, Präsident der DGRh und Leiter der Rheumaeinheit am Klinikum der LMU München zu bedenken. Das liege zum einen am entzündlichrheumatischen Geschehen selbst, denn auch durch ein aktiviertes Immunsystem kann die Infektabwehr geschwächt sein: Entzündungszellen, die sich gegen körpereigene Strukturen richten, verursachen die Rheumatypischen Symptome, fallen damit aber für die Infektabwehr aus. Zum anderen nehmen viele Rheumapatienten Medikamente, die die Immunabwehr unterdrücken, dies gilt insbesondere für Kortisonpräparate.

Menschen, die an einer entzündlich-rheumatischen Erkrankung leiden, sollten die von der DGRh und vom Robert-Koch-Institut veröffentlichten Hygiene- und Schutzmaßnahmen genau befolgen, um eine Infektion zu vermeiden. Wie andere Risikopatienten auch sollten Menschen mit Rheuma ihren Impfschutz überprüfen und in Abstimmung mit ihrem Rheumatologen vervollständigen. „Besonders die Impfungen gegen andere Atemwegserkrankungen wie Influenza, Pneumokokken und Keuchhusten sind derzeit wichtig", mahnt Schulze-Koops. Wichtig sei auch die sogenannte Umgebungsprophylaxe: Angehörige und enge Kontaktpersonen sollten prinzipiell auch über die von der Ständigen Impfkommission (STIKO) empfohlenen Schutzimpfungen verfügen.

Spezielle Vorsichtsmaßnahmen für Rheumapatienten:

- Auf keinen Fall ohne ärztlichen Rat die rheumatologische Basistherapie absetzen
- Ein Arzttermin zur Routineuntersuchung nach telefonischer Absprache möglicherweise verschieben

- Im Falle einer COVID-19-Erkrankung auch den behandelnden Rheumatologen (telefonisch) konsultieren

Besonders dringlich rät Schulze-Koops davon ab, aus Angst vor COVID-19 eigenmächtig die Rheumamedikation abzusetzen. „Hierfür gibt es bislang keinerlei Grundlage", betont er. Im Gegenteil sei bei einem plötzlichen Wegfall der Immunsuppression mit einem Wiederaufflammen der Rheuma-Symptomatik zu rechnen, welche aus oben genannten Gründen das Infektionsrisiko wiederum erhöhen kann. Wenn dann ein Rheumaschub nur mithilfe von Kortisonpräparaten in den Griff zu bekommen sei, steigt das Risiko, an einer Infektion zu erkranken noch stärker an. Rheumapatienten unter Immunsuppression, die im Gesundheitssystem oder anderen Bereichen arbeiten, in denen sie mit vielen Patienten in Kontakt kommen, müssen nicht vorsorglich und generell versetzt werden. Der übliche Infektionsschutz und das Einhalten der empfohlenen Maßnahmen ist Pflicht. Die individuelle Situation sollte mit dem Arbeitgeber besprochen werden.

Inwieweit diese Einschätzungen, die aus bereits bekannten auch viralen Infektionen abgeleitet sind, auch für das neuartige Coronavirus gelten, muss zwar abgewartet und immer wieder hinterfragt werden. Sobald es dazu neue Erkenntnisse gibt, wird sich die DGRh hierzu zeitnah äußern.

Der aktuelle Sachstand und aktuelle Empfehlungen online, unter: www.dgrh.de

\section{S. Priester, Pressestelle Deutsche Gesellschaft für Rheumatologie e.V.}


Hier steht eine Anzeige.

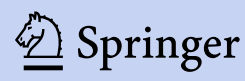

